\title{
A matrix formulation for noise transduction as a general case of noise measure
}

\author{
Hallgren, Robert B.
}

Published in:

IEEE Microwave and Guided Wave Letters

Link to article, DOI:

$10.1109 / 75.109128$

Publication date:

1992

Document Version

Publisher's PDF, also known as Version of record

Link back to DTU Orbit

Citation $(A P A)$ :

Hallgren, R. B. (1992). A matrix formulation for noise transduction as a general case of noise measure. IEEE Microwave and Guided Wave Letters, 2(1), 14-16. https://doi.org/10.1109/75.109128

\section{General rights}

Copyright and moral rights for the publications made accessible in the public portal are retained by the authors and/or other copyright owners and it is a condition of accessing publications that users recognise and abide by the legal requirements associated with these rights.

- Users may download and print one copy of any publication from the public portal for the purpose of private study or research.

- You may not further distribute the material or use it for any profit-making activity or commercial gain

- You may freely distribute the URL identifying the publication in the public portal

If you believe that this document breaches copyright please contact us providing details, and we will remove access to the work immediately and investigate your claim. 


\title{
A Matrix Formulation for Noise Transduction as a General Case of Noise Measure
}

\author{
Robert B. Hallgren, Member, IEEE
}

\begin{abstract}
Conventional noise characteristics of an active device are given by the minimum noise figure, $F_{\min }$, the optimum source reflection coefficient, $\Gamma_{\text {sopt }}$, and a noise resistance $R_{n}$. The noise measure extends the noise figure to include to available gain of network, for the case of a conjugate output match, and gives values that minimize the noise power available from the network consistent with maximum available gain. Noise transduction follows as a general case of noise measure by using the transducer gain with any output load. A new noise equation is derived that is solved for the minimum transduced noise, $N_{l}$, the optimum source impedance, and the optimum load impedance. These equations minimize the noise power delivered to the load impedance consistent with a maximum in the transducer gain.
\end{abstract}

\section{INTRODUCTION}

C ONVENTIONAL noise models for an active device or circuit use a noiseless two-port network and correlated noise sources [1]. The noise figure, as defined by Friis [2], is the ratio between the noise power originating from all the noise sources to the noise power originating from the generator noise source alone. This ratio has a minimum value for positive real source impedances, $F_{\min }$, and an associated optimum reflection coefficient $\Gamma_{\text {sopt }}$, as calculated from the network containing the noise sources only. Haus included the active network into the noise figure calculation and arrived at the noise measure equation [3]. The noise measure equation minimizes the noise power available from the network coincident with a maximum in the available gain [4].

The available noise power and the available gain are used in these equations so the values found are independent of the impedance at the output of the network. These values are used as a means to compare different devices, biases, or processes, and present a reasonable and convenient measure of device quality. In designs using the noise figure parameters, the choice of the output load is usually that value which gives the highest gain for the specified source impedance $z_{s}$. The optimum output load, $z_{\text {lopt }}$, is the conjugate of the output impedance of the network, $z_{\text {out }}$, with the optimum $z_{s}$ at the input.

Using $z_{\text {lopt }}$ is usually sufficient and gives the highest gain, but in cases where this impedance makes the network unstable, or where the gain of the network is low, a better choice for the load impedance is available. This report extends the noise measure equation to include the load impedance, and uses the noise power dissipated by the load impedance in place of the available noise power. The quantity found is thus the

Manuscript received August 12, 1991,

The author is with the Electromagnetics Institute, Technical University of Denmark, Building 348, DK 2800 Lyngby, Denmark.

IEEE Log Number 9105206.

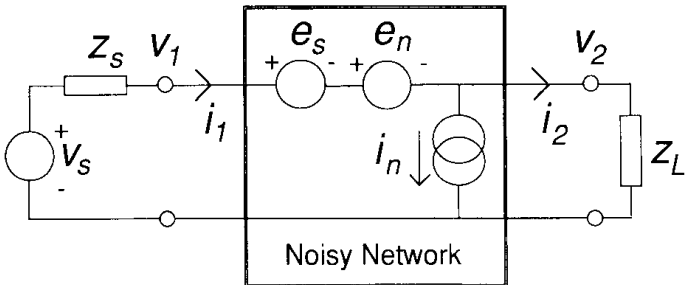

Fig. 1. Equivalent circuit used in noise figure calculations. All noise sources are included into a noisy network, which is driven by a noiseless source and terminated by a load impedance $Z_{l}$.

transduced noise, with the solution comprising the minimum noise transduced and both the source and the load impedances.

\section{NOISE Figures}

Fig. 1 shows the network used to calculate the noise figure. The noise of the network is modeled by the correlated chainform noise sources $e_{n}$ and $i_{n}$, with the the thermal noise of the source impedance $z_{s}$ modeled by $e_{s}$. All noise sources have been moved into an equivalent noisy network driven by a noiseless source and terminated by $z_{l}$. At the input:

$$
V_{1}=E_{s}+\delta+V_{2},
$$

where

$$
\begin{aligned}
V_{1}=\left(\begin{array}{c}
v_{1} \\
i_{1}
\end{array}\right), E_{s}=\left(\begin{array}{c}
e_{s} \\
0
\end{array}\right), \delta=\left(\begin{array}{c}
e_{n} \\
i_{n}
\end{array}\right), \\
\text { and } V_{2}=\left(\begin{array}{c}
v_{2} \\
i_{2}
\end{array}\right) .
\end{aligned}
$$

By defining an output impedance vector $Z_{l}^{\dagger}=\left(z_{l}^{*} 1\right)$, (where* denotes complex conjugation) the output vector is $V_{2}=i_{2} Z_{l}$. The source voltage is $v_{s}=v_{1}+i_{1} z_{s}$, or $v_{s}=Z_{s}^{\dagger} V_{1}$, with $Z_{s}^{\dagger} \triangleq\left(1 z_{s}\right)$. If $v_{s}=0$, premultiplying (1) by $Z_{s}^{\dagger}$ allows solving for the output current $i_{2}$. The autocorrelation of $i_{2}$ gives, assuming stationary random processes, the spectral density of the current at the output by the Wiener-Khintchine theorem [5]. For notational simplicity, terms such as $E_{s} E_{s}^{\dagger}$, for example, will represent the average of ensembles of spectra of windowed time functions of the source noise voltage generator output using single-sided spectra with $0 \leq \omega<\infty$.

Assuming independence between the thermal and the network sources, the autocorrelation of $i_{2}$ is

$$
\left\langle i_{2} i_{2}^{*}\right\rangle=\frac{Z_{s}^{\dagger} E_{s} E_{s}^{\dagger} Z_{s}+Z_{s}^{\dagger} \delta \delta^{\dagger} Z_{s}}{Z_{s}^{\dagger} Z_{l} Z_{l}^{\dagger} Z_{s}},
$$


where

$$
\delta \delta^{\dagger}=\left(\begin{array}{cc}
\left\langle e_{n} e_{n}^{*}\right\rangle & \left\langle e_{n} i_{n}^{*}\right\rangle \\
\left\langle i_{n} e_{n}^{*}\right\rangle & \left\langle i_{n} i_{n}^{*}\right\rangle
\end{array}\right)
$$

is the noise matrix. The matrix $P \triangleq\left(\begin{array}{ll}0 & 1 \\ 1 & 0\end{array}\right)$ will be used to allow writing $Z_{s}^{\dagger} P Z_{s}=2 \operatorname{Re}\left(z_{s}\right)$, giving the thermal noise voltage of the source as

$$
\begin{aligned}
Z_{s}^{\dagger} E_{s} E_{s}^{\dagger} Z_{s} & =4 k T \Delta f \operatorname{Re}\left(z_{s}\right) \\
& =2 k T \Delta f Z_{s}^{\dagger} P Z_{s} .
\end{aligned}
$$

with Boltzman constant $k$, the temperature $T$, and a bandwidth of $\Delta f$. The power dissipated by the load impedance is

$$
P_{\text {load }}=\left\langle i_{2} i_{2}^{*}\right\rangle \operatorname{Re}\left(z_{l}\right)=\frac{1}{2}\left\langle i_{2} i_{2}^{*}\right\rangle Z_{l} P Z_{l}^{\dagger}
$$

The noise figure uses the available noise power $P_{\text {avs }}=$ $P_{\text {load }}\left(z_{l}=z_{s}^{*}\right)$, which used a conjugate match at the network output. Since $Z_{s}^{\dagger} Z_{l}=\left(z_{l}+z_{s}\right)$, a conjugate load gives $Z_{s}^{\dagger} Z_{l}=2 \operatorname{Re}\left(z_{s}\right)$. Using $P$ makes $Z_{s}^{\dagger} Z_{l}=Z_{s}^{\dagger} P Z_{s}=Z_{s}^{\dagger} P Z_{l}$. Therefore, after substitution and cancellation in (6), the power available from the noise sources is

$$
P_{\text {avs }}=\frac{2 k T \Delta f Z_{s}^{\dagger} P Z_{s}+Z_{s}^{\dagger} \delta \delta^{\dagger} Z_{s}}{2 Z_{s}^{\dagger} P Z_{s}} .
$$

The noise figure for the network is the ratio of the noise power available from all sources, to the noise power available from the source impedance alone. The source impedance noise power is simply $k T \Delta f$, and from (7) the noise figure $F$ is

$$
F-1=\frac{Z_{s}^{\dagger} \delta \delta^{\dagger} Z_{s}}{2 k T \Delta f Z_{s}^{\dagger} P Z_{s}}
$$

The noise figure (8) is solved by finding the eigenvalue from the characteristic equation $\operatorname{det}\left(\lambda 1-P^{-1} \delta \delta^{\dagger}\right)=0$, where 1 is the identity matrix. Using $\lambda$ in (8) gives the corresponding eigenvector. The least-positive eigenvalue is $F_{\min }-1$, and the elements of its eigenvector give $z_{\text {sopt }}$ by division. For a singlesided spectrum $\left\langle e_{n} e_{n}^{*}\right\rangle=4 k T \Delta f R_{n}[6]$ and the conventional noise parameters are seen as simply a solution set of the noise figure eigenfunction (8).

\section{NoISE MEASURE}

The circuit used to find the noise measure is shown in Fig. 2. In addition to the noisy network, the active device or circuit is included as a two-port. The noise measure is found in a manner similar to that used to find the noise figure. The voltage and current at the input are the same as for the noise figure, but the transmission matrix, $T=\left(\begin{array}{ll}a & b \\ c & d\end{array}\right)$, is used to include the load impedance from the network output. The output current and voltage are related by $V_{l}=\left(\begin{array}{c}v_{l} \\ i_{l}\end{array}\right)=i\left(\begin{array}{c}z_{l} \\ 1\end{array}\right)$. At the active network input, $V_{2}=i_{l} T Z_{l}$, and from (1) the input to the noisy network is, thus, given by

$$
V_{1}=E_{s}+\delta+i_{l} T Z_{l} \text {. }
$$

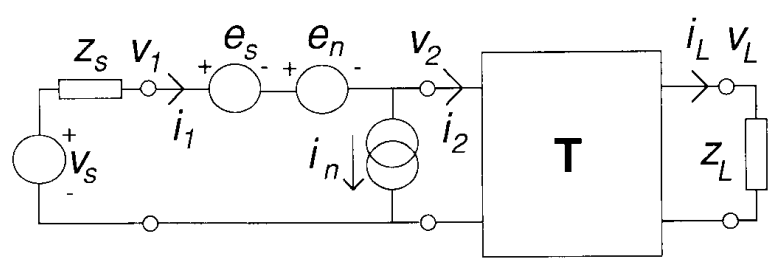

Fig. 2. Equivalent circuit used in noise measure and transduced noise calculations. Active network is added between the load and the noise network to include the available gain or the transducer gain into the noise equations.

With $v_{s}=0$, premultiplying (9) by $Z_{s}^{\dagger}$ gives $i_{l}$, with an autocorrelation of

$$
\left\langle i_{l} i_{l}^{*}\right\rangle=\frac{Z_{s}^{\dagger} E_{s} E_{s}^{\dagger} Z_{s}+Z_{s}^{\dagger} \delta \delta^{\dagger} Z_{s}}{Z_{s}^{\dagger} T Z_{l} Z_{l}^{\dagger} T^{\dagger} Z_{s}}
$$

The power dissipated by $z_{l}$ is $P_{\text {load }}=\frac{1}{2}\left\langle i_{l} i_{l}^{*}\right\rangle Z_{l}^{\dagger} P Z_{l}$, and the available output power is found using a conjugate match for $z_{l}$. With the network included in the circuit $z_{l}$ must be matched to the output impedance of the network $z_{\text {out }}$, including the source impedance $z_{s}$. The conjugate load can be found through a direct transformation of the source impedance vector via $Z_{s}^{\dagger} T=Z_{l}^{\dagger} P$, which gives $z_{l}^{*}=\frac{b+d z_{s}}{a+c z_{s}}$. This load impedance allows writing the denominator of $(10)$ as $\left(Z_{s}^{\dagger} T Z_{l}\right)\left(Z_{l}^{\dagger} T^{\dagger} Z_{s}\right)=\left(Z_{l}^{\dagger} P Z_{l}\right)\left(Z_{s}^{\dagger} T P T^{\dagger} Z_{s}\right)$. The noise power available from the network, $P_{\text {avn }}$, after multiplying (10) by $Z_{l}^{\dagger} P Z_{l}$, using a conjugate match in the denominator, and using (7), will be expressed by

$$
\begin{aligned}
\frac{P_{\mathrm{avn}}}{k T \Delta f} & =\left(\frac{2 k T \Delta f Z_{s}^{\dagger} P Z_{s}+Z_{s}^{\dagger} \delta \delta^{\dagger} Z_{s}}{2 k T \Delta f Z_{s}^{\dagger} P Z_{s}}\right) \cdot\left(\frac{Z_{s}^{\dagger} P Z_{s}}{Z_{s}^{\dagger} T P T^{\dagger} Z_{s}}\right) \\
& =F \cdot G_{a} .
\end{aligned}
$$

It gives the conventional noise figure (8) when divided by the available gain

$$
G_{a} \triangleq \frac{Z_{s}^{\dagger} P Z_{s}}{Z_{s}^{\dagger} T P T^{\dagger} Z_{s}}
$$

The noise measure as defined by Haus uses the quantity $N=\frac{G_{a}(F-1)}{G_{a}-1}$, which evaluates to

$$
N=\frac{Z_{s}^{\dagger} \delta \delta^{\dagger} Z_{s}}{2 k T \Delta f Z_{s}^{\dagger}\left(P-T P T^{\dagger}\right) Z_{s}^{\dagger}} .
$$

The noise measure equation (13) uses the increase in the network noise divided by the increase in the source noise, as measured at the network output. It is solved in a manner similar to the noise figure. The least-positive eigenvalue, as found from $\operatorname{det}\left(\lambda 1-\left[P-T P T^{\dagger}\right]^{-1} \delta \delta^{\dagger}\right)=0$, gives $N_{\min }$, and the corresponding eigenvalue gives $z_{\text {sopt }}$.

\section{NOISE Transduction}

The transduced noise begins with (10), and is a general case of a nonideal load impedance. If the source transformation is realized at the output, then the conjugate match will result in the lowest noise measure, at a maximum in both the available 
gain and in the transducer gain. If this transformation results in a load impedance that is unstable, or if other criteria must be met that requires a different load impedance, then the noise transduction can be used to optimize the transducer gain and minimize the noise power transduced to any load. Referring to (10) and Fig. 2, the power delivered to $z_{l}$ is $P_{\text {load }}=\frac{1}{2}\left\langle i_{l} i_{l}^{*}\right\rangle Z_{l}^{\dagger} P Z_{l}$. Substituting for $Z_{s}^{\dagger} E_{s} E_{s}^{\dagger} Z_{s}$ yields

$\frac{P_{\text {load }}}{k T \Delta f}=\left(\frac{2 k T \Delta f Z_{s}^{\dagger} P Z_{s}+Z_{s}^{\dagger} \delta \delta^{\dagger} Z_{s}}{2 k T \Delta f Z_{s}^{\dagger} P Z_{s}}\right) \cdot\left(\frac{Z_{l}^{\dagger} P Z_{l} Z_{s}^{\dagger} P Z_{s}}{Z_{s}^{\dagger} T Z_{l} Z_{l}^{\dagger} T^{\dagger} Z_{s}}\right)$

$=F \cdot G_{t}$

using the noise figure $F$ from (8), and the transducer gain defined by

$$
G_{t} \triangleq \frac{Z_{l}^{\dagger} P Z_{l} Z_{s}^{\dagger} P Z_{s}}{Z_{s}^{\dagger} T Z_{l} Z_{l}^{\dagger} T^{\dagger} Z_{s}}
$$

In a manner similar to that used for the noise measure, the noise transduction is obtained from (14) by using

$$
N_{t} \triangleq \frac{G_{t} \cdot(F-1)}{G_{t}-1}
$$

The denominator of (15) is expanded by noting $Z_{s}^{\dagger} T Z_{l} Z_{l}^{\dagger} T^{\dagger} Z_{s}$ $=Z_{l}^{\dagger} T^{\dagger} Z_{s} Z_{s}^{\dagger} T Z_{l}$, this is used to write the noise transduction (16) as two matrix equations, with one equation for each impedance vector. Written as an eigenfunction of $Z_{s}$, the noise transduced to the load is

$$
N_{t}=\frac{Z_{s}^{\dagger}\left[\delta \delta^{\dagger}\right] Z_{s}}{2 k T \Delta f Z_{s}^{\dagger}\left(P-\frac{T Z_{l} Z_{l}^{\dagger} T^{\dagger}}{2 \operatorname{Re}\left(z_{l}\right)}\right) Z_{s}}
$$

The least positive eigenvalue of (17) is the noise transduction, and the corresponding eigenvector is $Z_{s}$. The noise transduction as an eigenfunction for $Z_{l}$ is written as

$$
N_{t}=\left(\frac{Z_{s}^{\dagger} \delta \delta^{\dagger} Z_{s}}{4 k T \Delta f \operatorname{Re}\left(z_{s}\right)}\right) \frac{Z_{l}^{\dagger}[P] Z_{l}}{Z_{l}^{\dagger}\left(P-\frac{T^{\dagger} Z_{s} Z_{s} T^{\dagger}}{2 \operatorname{Re}\left(z_{s}\right)}\right) Z_{l}}
$$

The solutions to (18) are the load impedance vector and the noise transduction.

\section{CONCLUSION}

The conventional noise figure uses the available power from the noise sources and from the source impedance. The noise measure includes the available gain of the network and uses a load that is the conjugate of the network output impedance. The transduced noise allows for an arbitrary load and is a general case of the noise measure. Noise transduction uses the power delivered to the output load and thus depends upon both the input and the output impedances. Calculating the noise power transduced to the output results in a pair of matrix equations, the solutions to which are the input and output impedances and the transduced noise.

The noise transduction equations (17) and (18) reduce to the noise measure equation (13) under the condition of a matched load. With a matched load the transducer gain is the same as the available gain, and the values should agree. This equivalence requires that the optimum source impedance can be transformed exactly by the network and be realized as the output impedance. To find this load impedance from (13), the noise measure source impedance must be used, not the noise figure source impedance. For cases other than this the system will not be optimized. The source impedance that optimizes the system to a fixed load is found from (17). If the load is not fixed, but is otherwise restricted, then both (17) and (18) can be solved for the extrema of the noise transduced, as constrained to the range of $z_{l}$. This is perhaps the greatest utility of the transduced noise equations in that they allow finding an optimum impedance for both the source and the load of a device or circuit, for whatever range is available for the load. By including stability limits or interstage criteria, circuit or device noise performance can be optimized consistent with a maximum in the transducer gain.

\section{REFERENCES}

[1] R. Q. Twiss, "Nyquist's and Thevenin's theorem generalized for nonreciprocal linear networks," J. Appl. Phys., vol. 16, no. 5, pp. 599-602, May 1955.

[2] H. T. Friis, "Noise figures of radio receivers," Proc. IRE, vol. 32, no 7. pp. 419-422, July 1944.

[3] H. A. Haus, "Optimum noise performance of linear amplifiers," Proc. IRE, vol. 46, no. 8, pp. 1517-1539, Aug. 1958.

[4] H. A. Haus and R. B. Adler, Circuit Theory of Linear Noisy Networks. New York: Wiley, 1959

[5] E. Parzen, Stochastic Processes. Oakland, CA: Holden-Day, 1962

[6] H. Rothe and W. Dahlke, "Theory of noisy fourpoles," Proc. IRE, vol. 44 , no. 6 , pp. $811-818$, June 1956 